\title{
Monte Carlo filters for non-linear state estimation
}

\author{
Erik Bølviken ${ }^{1,2}$, Peter J. Acklam ${ }^{1,3}$,
} Nils Christophersen ${ }^{1}$ and John-Mikal Størdal ${ }^{3 *}$
(1) University of Oslo
(2) The Norwegian Computing Centre
(3) The Norwegian Defence Research Establishment

October 1, 1997

*Corresponding address: Erik Bølviken, University of Oslo, Institute of Mathematics, PO Box 1053 Blindern, N-0316 Oslo, Norway. 


\begin{abstract}
The application of Monte Carlo techniques to Bayesian state estimation is discussed. A simple theory for the Monte Carlo uncertainty is given and recursive Monte Carlo filters for general non-linear systems constructed from basic considerations. The methods are applied to a non-linear pendulum with measurement saturation and to bearings-only target tracking. The parameters of the measurement noise are in the bearings example determined on-line as part of the state estimation. The state vector then becomes six-dimensional, but the problem is still handled in real time. There is scope for improvement. Filter performance hinges on certain probability density estimates running in parallel with the filters. Errors in the estimated densities lead to filter inaccuracies that must be compensated by raising the number of Monte Carlo samples. Better ways of estimating the densities may lower this number and enhance speed.
\end{abstract}

Key words Extended Kalman filter, bootstrap filter, rejection filter, bearings-only tracking, measurement saturation. 


\section{Introduction}

Almost four decades after the publication of the Kalman filter, the problem of estimating the state of a non-linear, stochastic system from noisy observations is still open for research. The optimal solution is well known, but there has been no computational method that can handle general, multivariate systems within reasonable time limits. Monte Carlo techniques may in the future be able to play that role. We discuss in this paper recursive Monte Carlo filters that may approximate Bayesian estimates arbitrary well. Our framework is a non-linear, stochastic difference equation

$$
\begin{aligned}
& \mathbf{x}_{k}=\mathbf{f}_{k-1}\left(\mathbf{x}_{k-1}, \mathbf{u}_{k-1}, \mathbf{w}_{k-1}\right), k=1,2, \ldots ; \mathbf{x}_{0} \sim p\left(\mathbf{x}_{0}\right) \\
& \mathbf{z}_{k}=\mathbf{h}_{k}\left(\mathbf{x}_{k}, \mathbf{v}_{k}\right) .
\end{aligned}
$$

Here $\mathbf{x}_{k}$ is the state vector, $\mathbf{z}_{k}$ the vector of observations, $\mathbf{u}_{k}$ deterministic input, and $\mathbf{w}_{k}$ and $\mathbf{v}_{k}$ white noise processes, not necessarily additive. Define $\mathbf{Z}_{k}^{T}=\left\{\mathbf{z}_{1}^{T}, \mathbf{z}_{2}^{T}, \ldots, \mathbf{z}_{k}^{T}\right\}$ and let $p\left(\mathbf{x}_{k} \mid \mathbf{Z}_{k}\right)$ be the posterior density of $\mathbf{x}_{k}$ given $\mathbf{Z}_{k}$. Related notation, for example $p\left(\mathbf{x}_{k} \mid \mathbf{Z}_{k-1}\right)$ and $p\left(\mathbf{x}_{k} \mid \mathbf{x}_{k-1}\right)$ will also be needed, and the conditional densities of the measurements are denoted $p\left(\mathbf{z}_{k} \mid \mathbf{x}_{k}\right)$. The basic recursion connecting these quantities is

$$
\begin{aligned}
& p\left(\mathbf{x}_{k} \mid \mathbf{Z}_{k-1}\right)=\int_{-\infty}^{\infty} p\left(\mathbf{x}_{k} \mid \mathbf{x}_{k-1}\right) p\left(\mathbf{x}_{k-1} \mid \mathbf{Z}_{k-1}\right) d \mathbf{x}_{k-1}, \quad \mathbf{x}_{0} \sim p\left(\mathbf{x}_{0} \mid \mathbf{Z}_{0}\right)=p\left(\mathbf{x}_{0}\right) \\
& p\left(\mathbf{x}_{k} \mid \mathbf{Z}_{k}\right)=C\left(\mathbf{Z}_{k}\right) p\left(\mathbf{z}_{k} \mid \mathbf{x}_{k}\right) p\left(\mathbf{x}_{k} \mid \mathbf{Z}_{k-1}\right)
\end{aligned}
$$

where $1 / C\left(\mathbf{Z}_{k}\right)=p\left(\mathbf{z}_{k} \mid \mathbf{Z}_{k-1}\right)$ is a normalization constant. In the linear, Gaussian case the posterior densities are determined by their expectations and covariance matrices, which are provided by the Kalman filter. Solutions of (1.3) and (1.4) in general are only approximate. A useful distinction is between global and local techniques; see Kramer and Sorenson (1988). Examples of the former are the point mass method (Bucy and Senne, 1971), Gaussian sums (Alspach and Sorenson, 1972) and piecewise constant approximations (Kramer and Sorenson, 1988). Global methods evaluate the densities in (1.3) and (1.4) over a grid containing the main probability mass. Such computations grow fast with the dimension in $\mathbf{x}_{k}$ ("the curse of dimensionality'), and a cheaper, local method is often used, typically the extended Kalman filter.

Monte Carlo techniques also belong to the global type, but densities are represented as samples and evaluated by sampling. Realisations of $\mathbf{x}_{k}$ are then drawn randomly from the posterior density $p\left(\mathbf{x}_{k} \mid \mathbf{Z}_{k}\right)$. Their empirical mean approximates the Bayesian estimate $E\left(\mathbf{x}_{k} \mid \mathbf{Z}_{k}\right)$, and their variances yield assessments of uncertainty. The question is how to sample. Suppose samples of $\mathbf{x}_{0}$ have been generated. It is usually straightforward to pass them through the system equation and add realisations of system noise $\mathbf{w}_{0}$. This corresponds to the prediction step in Kalman filtering and yields samples from $p\left(\mathbf{x}_{1} \mid \mathbf{Z}_{0}\right)$. There is an additional update step, where the measurement $\mathbf{z}_{1}$ transforms the realisations from $p\left(\mathbf{x}_{1} \mid \mathbf{Z}_{0}\right)$ into realisations from $p\left(\mathbf{x}_{1} \mid \mathbf{Z}_{1}\right)$. The general Monte Carlo filtering problem is how to convert a collection of samples 
from $p\left(\mathbf{x}_{k-1} \mid \mathbf{Z}_{k-1}\right)$ into one from $p\left(\mathbf{x}_{k} \mid \mathbf{Z}_{k}\right)$. Methods of this kind were introduced in Müller (1991) and Gordon, Salmond and Smith (1993); see also West (1992), Gordon (1995) and Gordon, Salmond and Ewing (1995). The methodology is still in its infancy. The crucial issue is the update step. We argue in Section 3 that either of two algorithms from general sampling theory can be used. The so-called sampling importance resampling (SIR) scheme (Tanner, 1992) leads to the bootstrap filter with which most of the references above are concerned. Rejection sampling (Devroye, 1986) was used in Müller (1991) and has, in our view, important advantages. We construct in this paper a rejection filter from a perspective different from Müller's.

All these techniques must deal with a basic problem. To obtain $B$ samples of $\mathbf{x}_{k}$ with exactly the right distribution, more that $B$ realisations of $\mathbf{x}_{k-1}$ are needed. A high value of $B$ initially is not the answer. Reuse of some of the samples of $\mathbf{x}_{k-1}$ seems inevitable, but then $\mathbf{x}_{k}$ is drawn from a slightly wrong distribution. The error, which goes away as $B \rightarrow \infty$, will for finite $B$ aggravate over time, though likely to stabilize in the long run; see Section 4.1. A useful way to confront the issue is to use the samples of $\mathbf{x}_{k-1}$ to estimate its own posterior density. We may then sample from the estimate as many times as we please. The advantage of this viewpoint is that it opens up theory and methods from statistical density estimation for use in Monte Carlo filtering. Only the simplest of those methods have yet been tried out, but they do yield interesting results. A linear and non-linear pendulum is examined in Section 4. The performance of the Monte Carlo filter is compared to standard methods, and it is shown that the new method can deal with heavy measurement saturation. The second example (Section 5) is a problem from target tracking. Position and velocity of a moving target are to be determined from bearings-only information. The measurement noise comes from a first order low-pass filter, but is unknown otherwise. We show how noise parameters can be estimated on-line. This yields adaptive filters. The state vector for this problem is six-dimensional, but posterior distributions can still be computed by sampling.

Monte Carlo approximations to Bayesian estimates amounts to Monte Carlo integration, traditionally regarded as a slow numerical process. However, that picture changes if the uncertainty added by the Monte Carlo is compared to the uncertainty in the ordinary Bayesian estimate. In the next section a simple theory is developed, suggesting that 20-100 Monte Carlo replications may be enough. At present, inaccuracies in the filters force us to use higher numbers, but real time processing is still possible in examples such as the one in Section 5. The Monte Carlo approach has several other advantages. Parallelisation is easy, and there is no grid. Regions where the posterior densities are close to zero are avoided automatically, and high intercorrelation between state variables is actually a blessing, since the relevant part of the state space is shrinked. These points also applies to smoothing. It is possible to extend the Monte Carlo filtering in this direction, see Kitagawa (1996) who has designed an analogy to the fixed interval algorithm in Kalman filtering. Another approach is through one of the Markov chain Monte Carlo algorithms; see Carter and Kohn (1994), Shepard (1994), De Jong and Shepard (1995) and Fröwirth-Schnatter (1995) for applications to state estimation. 


\section{The Monte Carlo approach}

It is in this sequel convenient to remove time indices. Consider the problem of estimating $\mathbf{x}$ on the basis of a data vector $\mathbf{Z}$. The Bayesian solution is the conditional mean

$$
\hat{\mathbf{x}}=E(\mathbf{x} \mid \mathbf{Z}) .
$$

This estimate is optimal (minimum variance), but often intractable computationally. The Monte Carlo analogue is to sample $\mathbf{x}$ from its posterior distribution given $\mathbf{Z}$. Let $\mathbf{x}^{* b}, b=1, \ldots, B$, be such a Monte Carlo sample. (Superscript indices are used to separate a sequence of Monte Carlo samples from a time sequence. Symbols $b$ and $B$ stand for "bootstrap" following a convention from computational statistics.) The Monte Carlo average

$$
\overline{\mathbf{x}}^{* B}=B^{-1} \sum_{b=1}^{B} \mathbf{x}^{* b}
$$

may replace $\hat{\mathbf{x}}$ as estimator of $\mathbf{x}$. Obviously $\overline{\mathbf{x}}^{* B} \rightarrow \hat{\mathbf{x}}$ as $B \rightarrow \infty$, but the question is how large $B$ must be. To investigate this issue, let $P=E\left\{(\hat{\mathbf{x}}-\mathbf{x})(\hat{\mathbf{x}}-\mathbf{x})^{T}\right\}$ be the error covariance matrix of $\hat{\mathbf{x}}$ and $P^{B}=E\left\{\left(\overline{\mathbf{x}}^{* B}-\mathbf{x}\right)\left(\overline{\mathbf{x}}^{* B}-\mathbf{x}\right)^{T}\right\}$ the same quantity for the Monte Carlo estimate. We must expect the variances on the diagonal of $P^{B}$ to be larger than their counterparts in $P$, since they include Monte Carlo uncertainty in addition to problem specific variability. The following theorem is a precise description.

Theorem (i) The Monte Carlo estimate is unbiased, i.e. $E\left(\overline{\mathbf{x}}^{* B}-\mathbf{x}\right)=0$. (ii) Suppose the state vector $\mathbf{x}$ and the Monte Carlo samples $\mathbf{x}^{* b}$ are conditionally independent given $\mathbf{Z}$. Then $P^{B}=\left(1+B^{-1}\right) P$.

The proof is given in Appendix A. The condition in (ii), which expresses that randomness in the computer is unrelated to random noise in real life, is satisfied trivially. This assumption is basic for all the results in the present section. The theorem suggests that uncertainty due to sampling is small compared to uncertainty in the problem itself. For example, $B=20$ only adds an extra $5 \%$ to the variance. The view that a moderate value of $B$ is enough is a common one in computational statistics; see Efron and Tibshirani (1993).

The obvious estimate of uncertainty is

$$
P^{* B}=(B-1)^{-1} \sum_{b=1}^{B}\left(\mathbf{x}^{* b}-\overline{\mathbf{x}}^{* B}\right)\left(\mathbf{x}^{* b}-\overline{\mathbf{x}}^{* B}\right)^{T} .
$$

It is proved in Appendix A that

$$
E\left(P^{* B}\right)=\frac{B}{B+1} P^{B}
$$


The covariance matrix of the Monte Carlo samples is therefore an essentially unbiased estimate of the error covariance matrix of $\bar{x}^{* B}$. The variances of $P^{* B}$ may be compared to the variances of

$$
\hat{P}=E\left\{(\hat{\mathbf{x}}-\mathbf{x})(\hat{\mathbf{x}}-\mathbf{x})^{T} \mid \mathbf{Z}\right\},
$$

which would have been the obvious estimate of the error matrix $P=E\left\{(\hat{\mathbf{x}}-\mathbf{x})(\hat{\mathbf{x}}-\mathbf{x})^{T}\right\}$ had the computations been viable. For example, if $\hat{P}=\left\{\hat{P}_{i j}\right\}$ and $P=\left\{P_{i j}\right\}$, then $\hat{P}_{i j}$ is an unbiased, minimum variance estimate of $P_{i j}$. Let $\operatorname{var}(\hat{P})$ be the matrix $\left\{\operatorname{var}\left(\hat{P}_{i j}\right)\right\}$ and attach the same meaning to $\operatorname{var}\left(P^{* B}\right)$ and to other notation of a similar kind. Then

$$
\operatorname{var}\left(P^{* B}\right)=\operatorname{var}(\hat{P})+\frac{A_{1}}{B}+\frac{A_{2}}{B^{2}}, \quad A_{1}=E\left\{\operatorname{var}\left\{(\hat{\mathbf{x}}-\mathbf{x})(\hat{\mathbf{x}}-\mathbf{x})^{T} \mid \mathbf{Z}\right\}\right\},
$$

and $A_{2}$ is another constant matrix . A proof is given in Appendix A. To clarify the meaning of this formula, let $x$ be a scalar variable. Then $A_{1}=E \operatorname{var}\left\{(\hat{x}-x)^{2} \mid \mathbf{Z}\right\}$, which involves moments of order four. The normal case can be handled analytically. The variance estimate is then produced error-free by the Kalman filter (i.e. $\operatorname{var}(\hat{P})=0$ ), whereas it can be proved that $A_{1}=2\{\operatorname{var}(\hat{x}-x)\}^{2}$. The effect of Monte Carlo is therefore to induce a variance of magnitude approximately $2\{\operatorname{var}(\hat{x}-x)\}^{2} / B$.

\section{Monte Carlo filtering}

The Monte Carlo filtering problem is how to pass a sample $\mathbf{x}_{k-1}^{* b}, b=1, \ldots, B$ from $p\left(\mathbf{x}_{k-1} \mid \mathbf{Z}_{k-1}\right)$ on to one from $p\left(\mathbf{x}_{k} \mid \mathbf{Z}_{k}\right)$. Algorithms have a prediction and an update step, as in ordinary Kalman filtering. The update introduces a requirement that motivates the way the prediction step is carried out. Update is therefore discussed first.

\subsection{Monte Carlo measurement update}

Consider the update equation (1.4) and suppose it is possible to sample from the prediction density $p\left(\mathbf{x}_{k} \mid \mathbf{Z}_{k-1}\right)$. There are in the sampling literature two general algorithms that integrates the effect of the observation $\mathbf{z}_{k}$. Rejection sampling, outlined in Devroye (1986), assumes knowledge of a constant $M$ such that

$$
p\left(\mathbf{z}_{k} \mid \mathbf{x}_{k}\right) \leq M, \quad \text { for all } \mathbf{x}_{k} \text { and } \mathbf{z}_{k} .
$$

The conditional likelihood $p\left(\mathbf{z}_{k} \mid \mathbf{x}_{k}\right)$ is typically easy to compute, at least up to a constant of proportionality (which is enough). The following algorithm returns a random vector from $p\left(\mathbf{x}_{k} \mid \mathbf{Z}_{k}\right)$. 


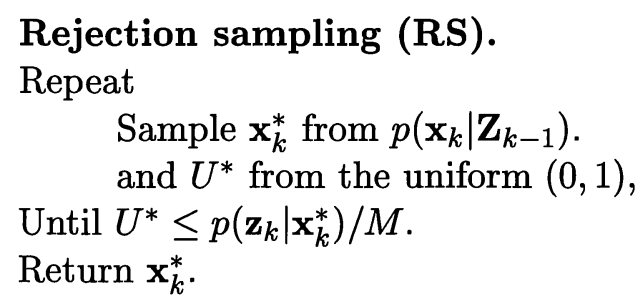

The stopping rule ensures that $\mathbf{x}_{k}^{*}$ on return has the right distribution although it originally came from a different one. An upper limit on the number of tries can not be stated in principle, but under good working conditions very many attempts is extremely unlikely; but see also Section 3.3. For high effciency $M$ in (3.1) should be as small as possible. When the algorithm is run $B$ times, an independent sample of size $B$ is obtained. Note that the normalisation constant $C\left(\mathbf{Z}_{k}\right)$ in (1.4) is not needed.

An alternative approximate technique is sampling importance resampling (SIR), see Tanner (1992). Here the $B$ realisations are generated jointly. Choose $B_{1} \gg B$ and go through the following steps:

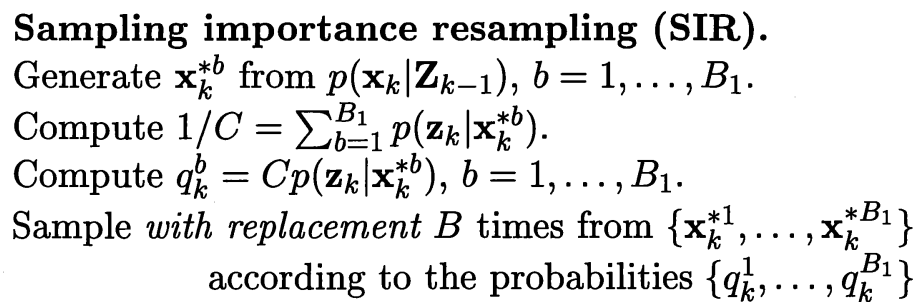

The scheme returns $B$ independent samples from $p\left(\mathbf{x}_{k} \mid \mathbf{Z}_{k}\right)$ as $B_{1} \rightarrow \infty$. For finite $B_{1}$ the algorithm is only approximate. This apart, SIR and RS work well under similar circumstances. $\mathrm{RS}$ is effective when the candidates are accepted quickly. The error in SIR is then small. It is suggested in Gordon et al. (1995) that $B_{1}$ should be at least $10 B$. Very efficient algorithms for sampling with replacement may be found in Devroye (1986).

\subsection{Monte Carlo prediction}

The second question is how to sample from the prediction density $p\left(\mathbf{x}_{k} \mid \mathbf{Z}_{k-1}\right)$ when $B$ realisations $\mathbf{x}_{k-1}^{* b}, b=1, \ldots, B$ from the preceding update density $p\left(\mathbf{x}_{k-1} \mid \mathbf{Z}_{k-1}\right)$ are at disposal. All of $\mathbf{x}_{k-1}^{* 1}, \ldots, \mathbf{x}_{k-1}^{* B}$ may be inserted into the system equation (1.1). When system noise is drawn, $B$ samples from $p\left(\mathbf{x}_{k} \mid \mathbf{Z}_{k-1}\right)$ are generated, but that is not enough. Both algorithms in Section 3.1 demanded more to produce $B$ independent samples from the update distribution. Gordon et al. (1993) therefore suggested reuse. They specifically proposed to sample with replacement the required number of times from the set $\left\{\mathbf{x}_{k-1}^{* 1}, \ldots, \mathbf{x}_{k-1}^{* B}\right\}$. Such a scheme may work when there is enough system noise. The discrete set of values from $\left\{\mathbf{x}_{k-1}^{* 1}, \ldots, \mathbf{x}_{k-1}^{* B}\right\}$ are then spread out when taken through the system. However, with no system noise the number of different values 
in the sample is bound to be reduced as the filter progresses, even to the point of collapsing to a single value. The problem was in Gordon et al. (1993) alleviated by a so-called 'roughening' technique. A random jitter was added each updated simulation and combined with an editing procedure where samples with small normalised likelihoods were removed; see also Müller (1991).

The issue is in our view put on a more rational footing if the samples from $p\left(\mathbf{x}_{k-1} \mid \mathbf{Z}_{k-1}\right)$ are used to estimate its own density. The samples needed can then be drawn the estimated distribution. An advantage of this viewpoint is that methods and theory from a highly developed area of statistics become available; see Silverman (1986) and Scott (1992) for reviews on density estimation. Some possibilities are mentioned in Section 6. Only standard kernel density estimates have been used here. This coincides with suggestions in West (1992) and Gordon (1995). The following procedure resembles the practice of adding artificial system noise in Kalman filtering to keep the filter 'open' to new measurements, although in the present case variance is not increased.

Let $\overline{\mathbf{x}}_{k-1}^{* B}$ be the mean vector and $P_{k-1}^{* B}$ the covariance matrix of the Monte Carlo samples $x_{k-1}^{* b}$, $b=1, \ldots, B$. Consider the distribution that assigns probabilities $1 / B$ to each of

$$
\overline{\mathbf{x}}_{k-1}^{* B}+\left\{\left(1-\zeta^{2}\right) B /(B-1)\right\}^{1 / 2}\left(\mathbf{x}_{k-1}^{* b}-\overline{\mathbf{x}}_{k-1}^{* B}\right), \quad b=1,2, \ldots, B .
$$

Here $\zeta \in[0,1]$ is user selected. It is easy to verify that the distribution has mean $\overline{\mathbf{x}}_{k-1}^{* B}$ and covariance matrix $\left(1-\zeta^{2}\right) P_{k-1}^{* B}$. Kernel density estimates of $p\left(\mathbf{x}_{k-1} \mid \mathbf{Z}_{k-1}\right)$ are convolutions of this discrete distribution with a freely chosen kernel distribution $K_{\zeta}$; for example a Gaussian. The convolution should have the same mean $\overline{\mathbf{x}}_{k-1}^{* B}$ and covariance matrix $P_{k-1}^{* B}$ as the sample. This is ensured if $K_{\zeta}$ has mean zero and covariance matrix $\zeta^{2} P_{k-1}^{* B}$. Sampling is easy. We draw from the discrete distribution (3.2) and add a random variable generated from $K_{\zeta}$. The sum, approximately distributed as $p\left(\mathbf{x}_{k-1} \mid \mathbf{Z}_{k-1}\right)$, is inserted into the system equation (1.1) and yields when system noise is drawn, a realisation from an estimate of the prediction density. In detail the algorithm runs as follows:

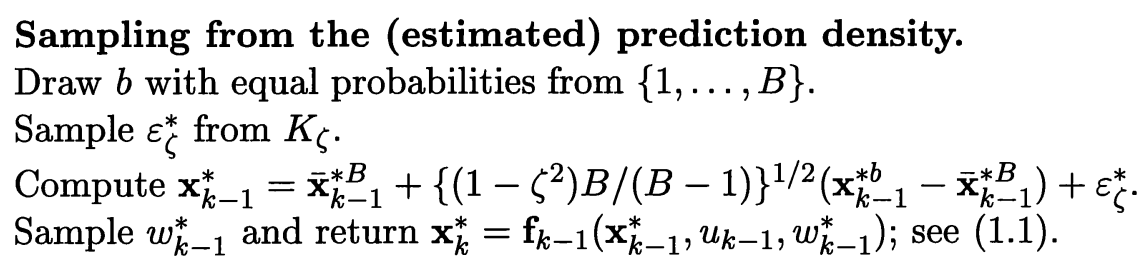

The choice of $\zeta$ is much discussed in the literature on density estimation; see Silverman (1986).

\subsection{Main algorithm}

Monte Carlo filters are syntheses of the algorithms in the preceding sections. Rejection sampling leads to 


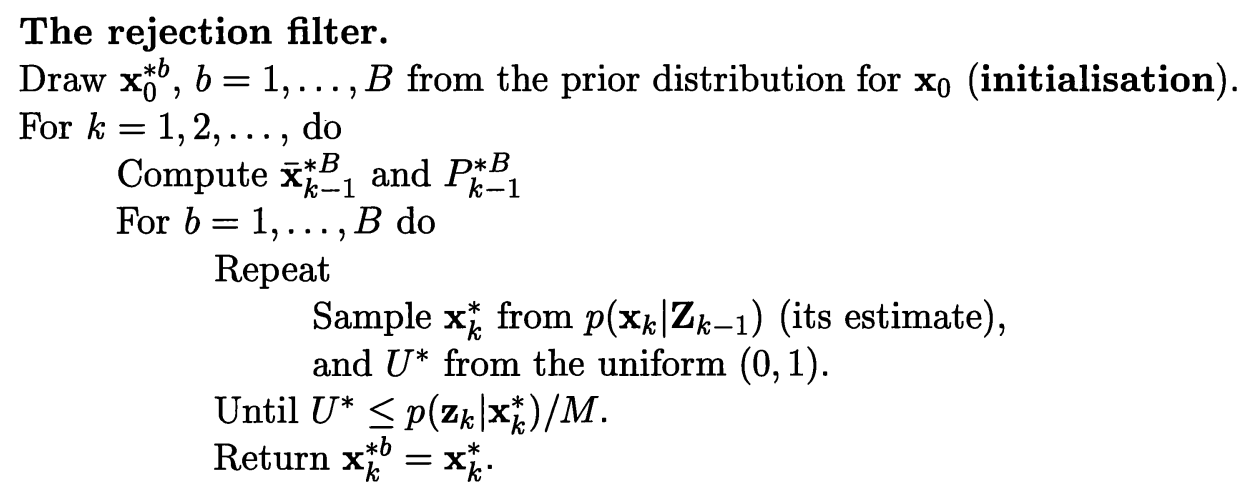

Use of the SIR algorithm is similar and leads to the bootstrap filter in Gordon et al. (1993). Calculation of the upper bound $M$ in (3.1) is then avoided. The rejection filter has a forerunner in Müller (1991).

The examples in Sections 4 and 5 have been run with the rejection filter. When the bootstrap filter was tried as well, it usually gave comparable results. The principal difference between the two methods lies in the number of candidates drawn from $p\left(\mathbf{x}_{k} \mid \mathbf{Z}_{k-1}\right)$ (the estimate). Let $R_{k}$ be the average number needed to generate one sample from the update density $p\left(\mathbf{x}_{k} \mid \mathbf{Z}_{k}\right)$. For the bootstrap filter $R_{k}$ is fixed at some $r_{0}$ (say ten), but for the rejection filter $R_{k}$ varies randomly. There are sound arguments in favour of the latter strategy, provided a suitable $M$ can be found. First, recall that random $R_{k}$ is the consequence of using the rejection sampling algorithm (Section 3.1) which is exact. Error due to SIR only being approximate is then avoided. Secondly, a major part of the numerical work is the generation of the candidates. It seems computationally wasteful to fix $R_{k}$ in advance when experience dictates that $R_{k}$ often is much smaller that $r_{0}$. An even more important point is that random allocation of $R_{k}$ tends to stabilize the method. Chance effects occasionally cause the filter to have trouble in finding candidates to match a new measurement. This may happen during a completely normal run and is due to accumulation of error in the density estimates. To allow the filter to work long to find the right samples corrects the error and prevents divergence. Almost always, a small $R_{k}$ is enough at the very next point in time, and the filter might be able to catch up with the measurements should it have fallen behind.

\section{A simple pendulum}

The performance of the rejection filter will in this section be examined against established methods. We shall also demonstrate the ability of the new method to deal with heavy measurement saturation. The example is an undamped pendulum with angular position $x_{1}(t)$ and angular velocity $x_{2}(t)$. The system model is

$$
\dot{x}_{1}(t)=x_{2}(t), \quad \dot{x}_{2}(t)=-\frac{g}{L} \sin \left\{x_{1}(t)\right\}+w(t),
$$


where $g=9.81 \mathrm{~m} / \mathrm{s}^{2}$ is the gravitational acceleration, $L=2 \mathrm{~m}$ the rod length and $w(t)$ Gaussian, white noise (continuous). The period of the linearized pendulum, where $\sin \left\{x_{1}(t)\right\}$ is approximated with $x_{1}(t)$, is about $2.2 \mathrm{~s}$. Angular position was observed at $t_{k}=k \delta$ according to $z_{k}=x_{1 k}+v\left(t_{k}\right)$, where $x_{1 k}=x_{1}\left(t_{k}\right)$. The sampling rate was $\delta=0.25 \mathrm{~s}$ and measurement noise Gaussian and white (discrete). Let $\sigma$ be the standard deviation. The conditional likelihood $p\left(z_{k} \mid \mathbf{x}_{k}\right)$ is then bounded above by $M=(2 \pi)^{-1 / 2} \sigma^{-1}$, see (3.1). Thus

$$
\frac{p\left(z_{k} \mid \mathbf{x}_{k}\right)}{M}=\exp \left\{-\frac{1}{2 \sigma^{2}}\left(z_{k}-x_{1 k}\right)^{2}\right\},
$$

which is needed for the update step of the rejection filter. The discrete approximation to (4.1), required for the prediction step, was found by using a step length of $0.05 \mathrm{~s}$.
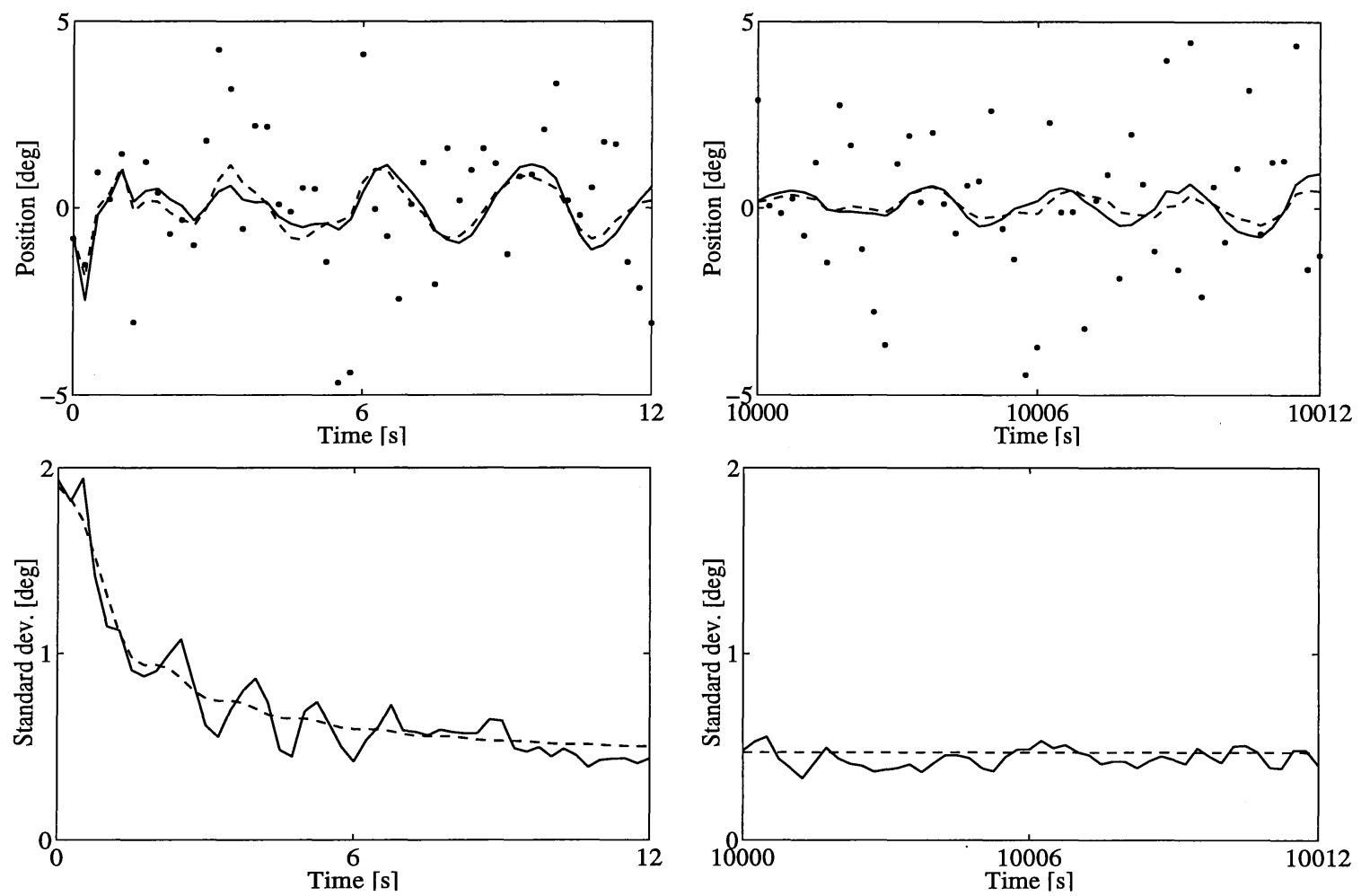

Figure 1: The linear pendulum tracked by the rejection and Kalman filters. Error in estimated angular position (upper row) and their estimated standard deviation (lower row). Initial phase to the left and steady state to the right. Solid line: rejection filter, dashed line: Kalman filter, dots: observations. 


\subsection{Linear pendulum}

The first round of experiments was run on the linear approximation to (4.1). Results from the Monte Carlo and Kalman filters are compared. The square root of the spectral density was $0.5^{\circ} / \mathrm{s}^{3 / 2}$ for the system noise (angular velocity only) and the standard deviation was $2^{\circ}$ for the measurement noise. The pendulum started at zero angle with angular velocity $12 \%$. In the filters these initial states were assigned Gaussian prior distributions with expectations zero and standard deviations $6^{\circ}$ (angular position) and $18^{\circ}$ s (speed). All experiments are run with $B=100$ Monte Carlo replications. The density estimate (Section 3.2) used a Gaussian kernel density and smoothing parameter $\zeta=0.4$.

Figure 1 shows errors in angular position as estimated by the ordinary Kalman filter and by the rejection filter. Their (estimated) standard deviations are compared in the second row. The results produced by the two methods are similar, and would have looked identical had the Monte Carlo filter been run with a higher sample size. The figures on the right (after 40000 observations) demonstrate that the new filter has stable performance in the long run. Indeed, the vectorial stochastic process $\left(\mathbf{x}_{k}, \mathbf{x}_{k}^{* 1}, \ldots, \mathbf{x}_{k}^{* B}\right)$, defined by augmenting the real state vector and the Monte Carlo samples, is a Markov process that will reach equilibrium as $k \rightarrow \infty$ under suitable, albeit presently unknown, mathematical conditions. The estimates of the standard deviation provided by the rejection filter are slight underestimates for reasons discussed in Section 6. Estimates of angular velocity from the two filters were similar and confidence bounds obtained from posterior samples (see next section) were nicely placed around the true values.
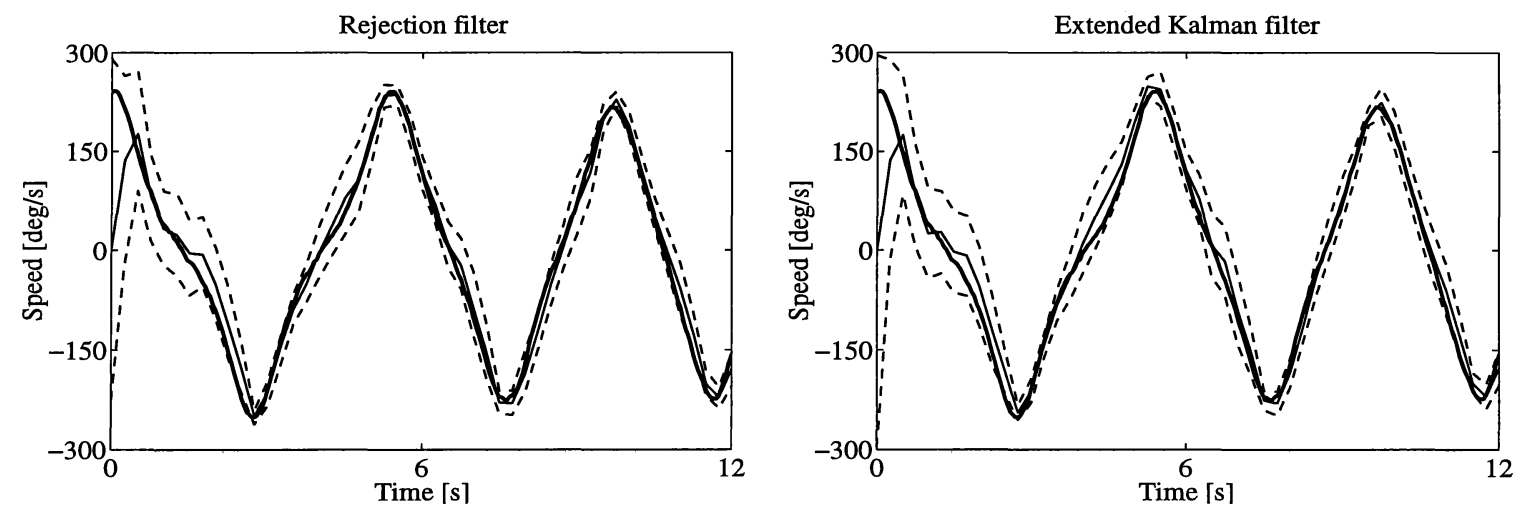

Figure 2: True and estimated angular velocity for the non-linear pendulum using the rejection filter (left) and the extended Kalman filter (right). Thick solid line: true value, thin solid line: filtered value, dashed lines: $90 \%$ confidence bounds.

\subsection{Non-linear pendulum}

The second round of experiments were carried out on the non-linear pendulum. The square root of the spectral density of the system noise was now $10^{\circ} / \mathrm{s}^{3 / 2}$ and the standard deviation of 
the measurement noise $20^{\circ}$. The pendulum started at angular position zero with angular speed $240 \%$ s. The prior distribution for the initial state was Gaussian with mean zero and standard deviations $60^{\circ}$ (position) and $180^{\circ} / \mathrm{s}$ (angular speed). This means that little was known about the initial state in advance. The rejection filter was run under the technical conditions in Section 4.1.

An implementation of the extended Kalman filter has in Figure 2 been compared to the Monte Carlo filter. Note that the state variable displayed is angular speed. The agreement between the two methods is very high (and was even higher for position). This also applies to the 90\% confidence bounds. For the Monte Carlo filters interval estimates can be constructed from the posterior samples. Consider angular speed $x_{2 k}=x_{2}\left(t_{k}\right)$. For a $90 \%$ interval based on $x_{2 k}^{* 1}, \ldots, \mathbf{x}_{2 k}^{* B}$, let $l=0.05 B$ and take as lower and upper confidence limits the $l$ 'th smallest and the $l$ 'th largest of these samples.

Measurement saturation is introduced in Figures 3 and 4. In the first of these experiments
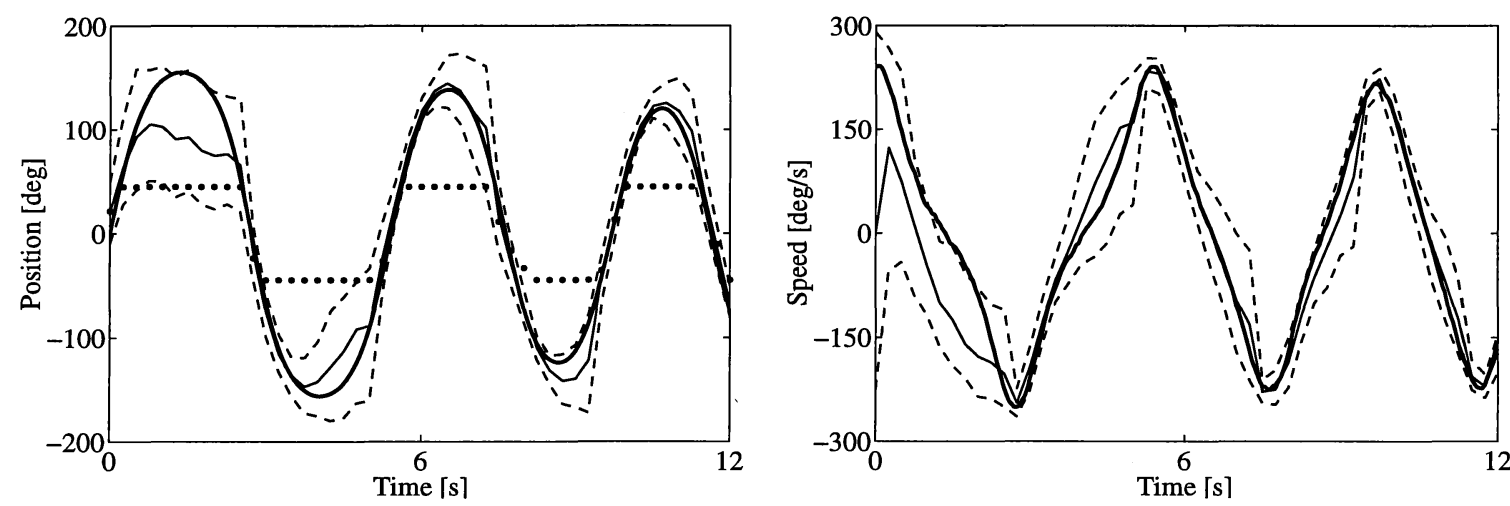

Figure 3: Rejection filter estimates of position (left) and angular velocity (right) for the nonlinear pendulum with measurement saturation at $\pm 45^{\circ}$. Thick solid line: true value, thin solid line: filtered value, dashed lines: $90 \%$ confidence limits, dots: observations.

(Figure 3) the sensor was unable to follow pendulum movements beyond $\pm 45^{\circ}$. It is not obvious how such information should be utilized with the extended Kalman filter, but easy with the rejection filter which only requires an appropriate respecification of the probability density function $p\left(z_{k} \mid \mathbf{x}_{k}\right)$; see Section 3. Implementation requires little work. It is enough to change a few statements in the program without measurement saturation. The rejection filter is even able to follow the pendulum movements with saturation at $0^{\circ}$ (Figure 4 ). This means that the sensor only records whether angular position is below or above zero. The confidence limits are now wider than in Figure 3, as is plausible. Not much was known about the initial state of the pendulum in advance, but knowledge of the underlying physical model is enough to determine them even from observations as poor as in Figure 4. 

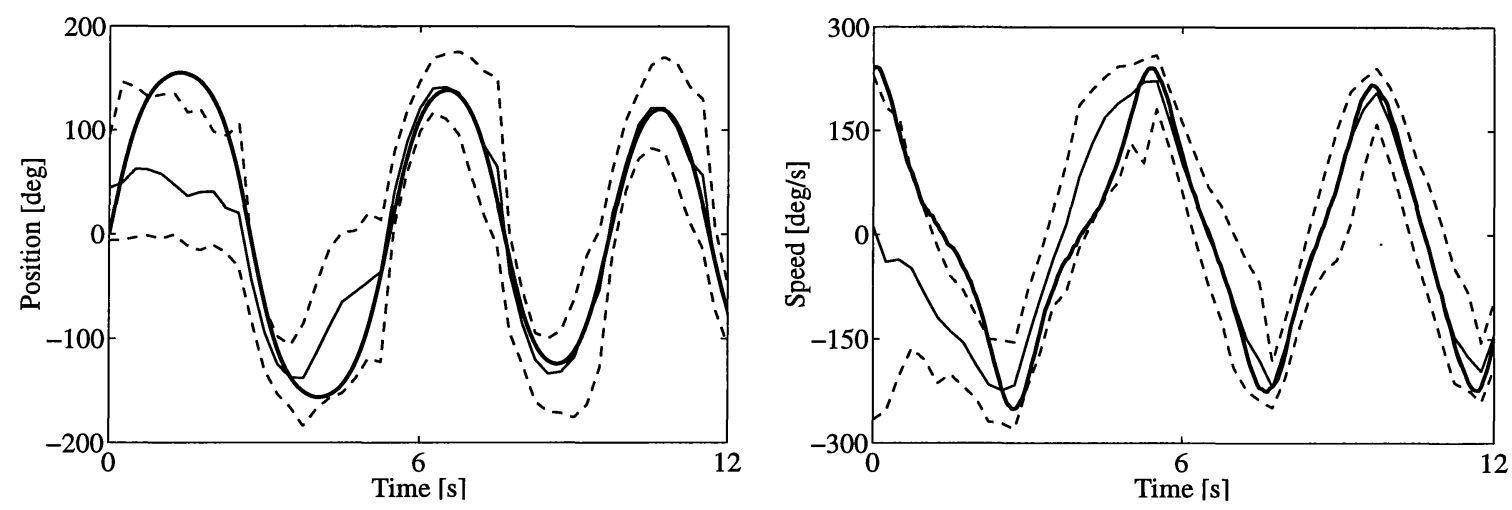

Figure 4: Rejection filter estimates of position (left) and angular velocity (right) when measuring only the sign. Thick solid line: true value, thin solid line: filtered value, dashed lines: $90 \%$ confidence limits.

\section{Bearings-only tracking}

\subsection{Problem and model}

The second example has been much discussed in the tracking literature; see Borstad (1985). An object, moving at constant velocity, is observed through noisy bearing measurements by an observer wanting to determine position and velocity as quickly as possible and continue to track the object. To make the problem identifiable, the observer must himself move. The set-up is displayed in Figure 5. The observer follows a zigzag curve (lower, left corner) and the target the solid straight line.

The state vector $\mathbf{x}_{k}=\left(x_{1 k}, \ldots, x_{4 k}\right)^{T}$ is four-dimensional, consisting at time $t_{k}=k \delta$ of position coordinates $\left(x_{1 k}, x_{2 k}\right)$ and velocity coordinates $\left(x_{3 k}, x_{4 k}\right)$. The system model is for position $x_{1 k}=x_{1 k-1}+\delta x_{3 k-1}$ and $x_{2 k}=x_{2 k-1}+\delta x_{4 k-1}$ and for velocity $x_{3 k}=x_{3 k-1}$ and $x_{4 k}=x_{4 k-1}$. There is no system noise. Suppose the observer at time $t_{k}$ is at $\left(o_{1 k}, o_{2 k}\right)$. The measurement is then

$$
z_{k}=\mu\left(\mathbf{x}_{k}\right)+v_{k}, \quad \text { where } \quad \mu\left(\mathbf{x}_{k}\right)=\operatorname{atan}\left(\frac{x_{1 k}-o_{1 k}}{x_{2 k}-o_{2 k}}\right) .
$$

Here $z_{k}$ is the angle with the vertical axis. The coordinates $\left(o_{1 k}, o_{2 k}\right)$ are, of course, known to the observer. The challenge when trying to estimate $\mathbf{x}_{k}$ is that angular measurements are not as informative as observing distances and positions directly. Moreover, the measurement equation is strongly non-linear. Another problem is that the parameters of the measurement noise may vary with the distance between observer and target and also with daily conditions. Noise is often assumed to have been generated by a low-pass filter. A first approximation in discrete time is

$$
v_{k}=\alpha v_{k-1}+\eta_{k-1}
$$


where $0 \leq \alpha \leq 1$ and $\eta_{k}$ is white, Gaussian noise with standard deviation $\sigma$. The method developed determines $\alpha$ and $\sigma$ on-line, jointly with the estimation of the state vector $\mathbf{x}_{k}$. The bootstrap filter was applied to this problem in Gordon, et al. (1993) and also in Gordon (1995) and in Gordon, et al. (1995), but none of these references dealt with coloured noise and adaptive estimation of noise parameters.

\subsection{Adaptive filters.}

We shall identify $\sigma$ with a fifth state variable and $\alpha$ with a sixth one. It is straightforward to write down the system model, but the conditional likelihoods needed by the rejection filter are less obvious, and raise several issues. First, suppose that the noise $v_{k}$ is white (i.e. $\alpha=0$ ). The Gaussian likelihoods then become

$$
p\left(z_{k} \mid \mathbf{x}_{k}, \sigma\right)=(2 \pi)^{-1 / 2} \sigma^{-1} \exp \left\{-\frac{1}{2 \sigma^{2}}\left(z_{k}-\mu\left(\mathbf{x}_{k}\right)\right)^{2}\right\} .
$$

One effect of defining $\sigma$ as a fifth state variable is that the maximum over all $z_{k}$, $\mathbf{x}_{k}$ and $\sigma$, i.e., $M$ in (3.1), becomes infinite (take $z_{k}=\mu\left(\mathbf{x}_{k}\right)$ and let $\sigma \rightarrow 0$ ). This would stall the rejection filter completely, but a simple remedy is to introduce a lower, positive limit for $\sigma$. Suppose the prior distribution for $\sigma$ has all probability mass concentrated above $\sigma_{0}$. Then $M=(2 \pi)^{-1 / 2} \sigma_{0}^{-1}$ and

$$
\frac{p\left(z_{k} \mid \mathbf{x}_{k}, \sigma\right)}{M}=\frac{\sigma_{0}}{\sigma} \exp \left\{-\frac{1}{2 \sigma^{2}}\left(z_{k}-\mu\left(\mathbf{x}_{k}\right)\right)^{2}\right\} .
$$

It is advisable to define the fifth state variable as a non-linear transformation of $\sigma$. There are several possibilites. We have chosen

$$
x_{5}=\log \left(\sigma-\sigma_{0}\right),
$$

which maps $\sigma$ on to the whole real line. The operation is likely to bring the posterior distributions closer to a symmetric one, and this may improve the performance of density estimates based on Gaussian kernels (Section 3.2). Another advantage is that whatever the value sampled for $x_{5}$, the inverse mapping $\sigma=\sigma_{0}+\exp \left(x_{5}\right)$ guarantees $\sigma$ to be within the admissible region. Formally $x_{5 k}=x_{5 k-1}$ is added as a fifth system equation, and the state vector is expanded into $\mathbf{x}_{k}=\left(x_{1 k}, \ldots, x_{5 k}\right)^{T}$. When $\sigma$ is replaced by $x_{5 k}$ in (5.3), we obtain

$$
\frac{p\left(z_{k} \mid \mathbf{x}_{k}\right)}{M}=\left(1+\frac{\exp \left(x_{5 k}\right)}{\sigma_{0}}\right)^{-1} \exp \left(-\frac{\left\{z_{k}-\mu\left(\mathbf{x}_{k}\right)\right\}^{2}}{2\left\{\sigma_{0}+\exp \left(x_{5 k}\right)\right\}^{2}}\right) \text {. }
$$

As in ordinary Kalman filtering, correlated measurement noise presents an additional problem. The update equation (1.4) is now valid only in the more general form

$$
p\left(\mathbf{x}_{k} \mid \mathbf{Z}_{k}\right)=C\left(\mathbf{Z}_{k}\right) p\left(\mathbf{z}_{k} \mid \mathbf{x}_{k}, \mathbf{Z}_{k-1}\right) p\left(\mathbf{x}_{k} \mid \mathbf{Z}_{k-1}\right) \quad \text { (Bayes' formula). }
$$


Note that the conditional likelihood for $\mathbf{z}_{k}$ depends on earlier measurements in addition to $\mathbf{x}_{k}$. This means that the more complicated density function $p\left(\mathbf{z}_{k} \mid \mathbf{x}_{k}, \mathbf{Z}_{k-1}\right)$ takes the place of the simpler $p\left(\mathbf{z}_{k} \mid \mathbf{x}_{k}\right)$ in the algorithms in Section 3. Work is in progress to accomodate coloured noise in recursive Monte Carlo filters (Eidsvik, 1997). In the present case without system noise, a simplified approach is possible. From (5.1) and (5.2) we obtain

$$
z_{k}=\alpha z_{k-1}+\mu\left(\mathbf{x}_{k}\right)-\alpha \mu\left(\mathbf{x}_{k-1}\right)+\eta_{k-1} .
$$

Hence

$$
p\left(z_{k} \mid \mathbf{x}_{k}, \sigma, \alpha, \mathbf{Z}_{k-1}\right)=(2 \pi)^{-1 / 2} \sigma^{-1} \exp \left\{-\frac{1}{2 \sigma^{2}}\left(z_{k}-\alpha z_{k-1}-\mu\left(\mathbf{x}_{k}\right)+\alpha \mu\left(\mathbf{x}_{k-1}\right)\right)^{2}\right\}
$$

and, similar to (5.3)

$$
\frac{p\left(z_{k} \mid \mathbf{x}_{k}, \sigma, \alpha, \mathbf{Z}_{k-1}\right)}{M}=\frac{\sigma_{0}}{\sigma} \exp \left\{-\frac{1}{2 \sigma^{2}}\left(z_{k}-\alpha z_{k-1}-\mu\left(\mathbf{x}_{k}\right)+\alpha \mu\left(\mathbf{x}_{k-1}\right)\right)^{2}\right\} .
$$

Note that this is valid only because $\mathbf{x}_{k-1}$ is uniquely determined by $\mathbf{x}_{k}$. Two extra state variables are now needed. As the fifth one take (5.4) and as the sixth introduce

$$
x_{6}=\log \left(\frac{\alpha}{1-\alpha}\right) .
$$

The rational for this transformation is the same as for (5.4). For example, $x_{6}$ extends over the whole real line if $\alpha$ varies between 0 and 1. The state vector is now $\mathbf{x}_{k}=\left(x_{1 k}, \ldots, x_{6 k}\right)^{T}$ and the sixth system equation $x_{6 k}=x_{6 k-1}$. It is easy to verify that

$$
\frac{p\left(z_{k} \mid \mathbf{x}_{k}, \mathbf{Z}_{k-1}\right)}{M}=\left(1+\frac{\exp \left(x_{5 k}\right)}{\sigma_{0}}\right)^{-1} \exp \left(\frac{\left\{z_{k}-\alpha_{k} z_{k-1}-\mu\left(\mathbf{x}_{k}\right)+\alpha_{k} \mu\left(\mathbf{x}_{k-1}\right)\right\}^{2}}{2\left\{\sigma_{0}+\exp \left(x_{5 k}\right)\right\}^{2}}\right),
$$

where $\alpha_{k}=\exp \left(x_{6 k}\right) /\left(1+\exp \left(x_{6 k}\right)\right)$, which is the inverse of (5.8).

\subsection{Experiments}

The performance of filters for bearings-only tracking depends on the movements of target and observer and on their relative distance. Estimates given here become optimal (unbiased and minimum variance) as $B \rightarrow \infty$. In practice little change was experienced when $B$ was raised above 5000. The estimates for $B=5000$ are therefore close to the optimal ones. To prevent divergence phenomena we had to use higher values for $B$ than in Section 4 . Results for $B=1000$ and $B=5000$ are reported below. Neither choice gives an excessive computational load. Our (non-optimized) fortran code ran twice as fast as real time on an (old) SG Indy computer $(100 \mathrm{Mz}$, Mips R4610, cache size $16 \mathrm{kB})$ when $B=5000$ and ten times as fast when $B=1000$. 

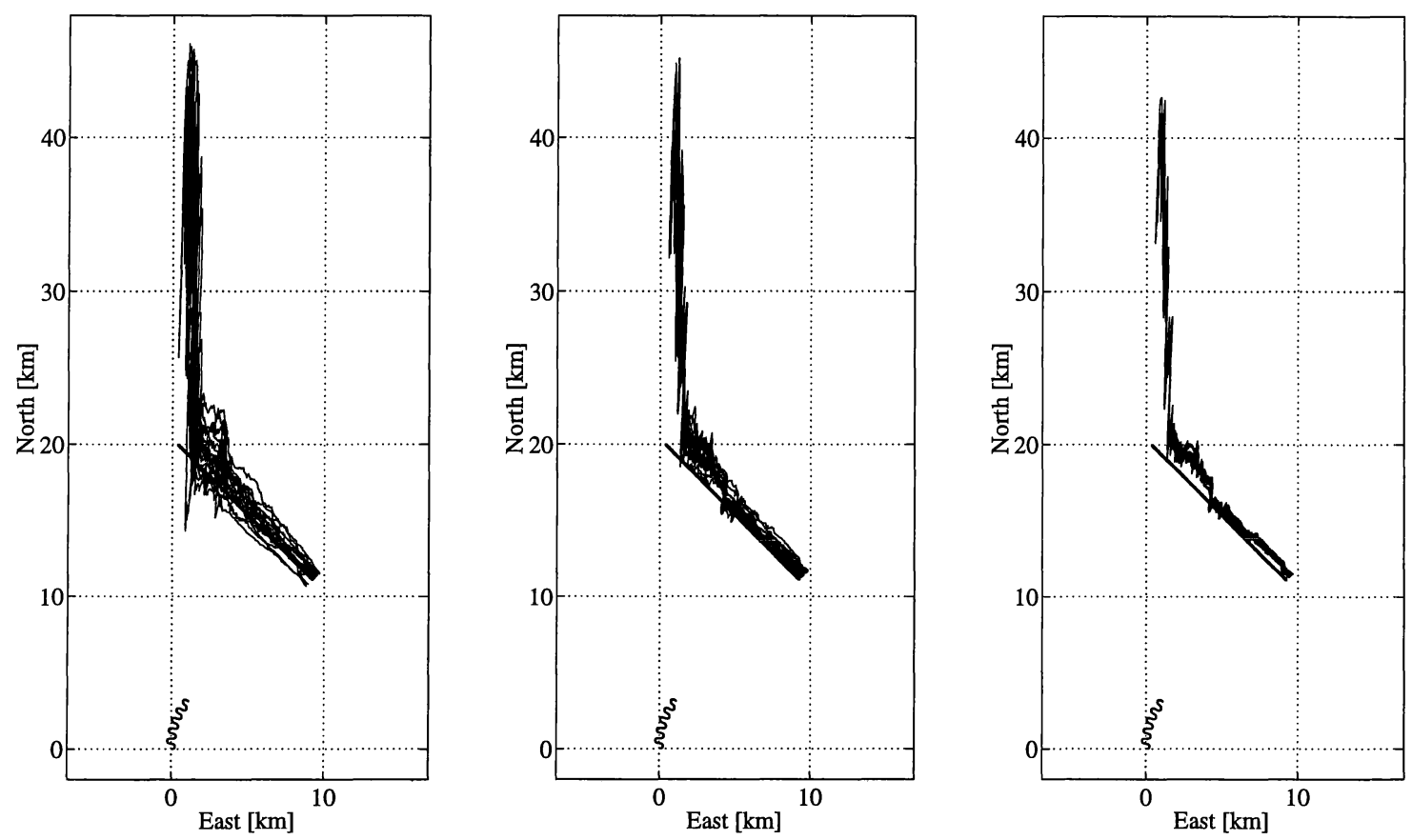

Figure 5: Repeated, filtered estimates of position (fixed scenario) for white measurement noise $\left(\alpha=0\right.$ and $\left.\sigma=0.3^{\circ}\right)$. Twenty different observation sequences to the left and twenty identical ones in the middle and to the right. Monte Carlo samples: 1000 to the left and in the middle, 5000 to the right.

The experiments were run on simulated data on a set-up as shown in Figure 5. The observer moved in the zigzag pattern in the lower left corner with speed $4 \mathrm{~m} / \mathrm{s}$. Unbiased, angular measurements were taken every five seconds with Gaussian noise described by the model in (5.2). The parameters $\alpha$ and $\sigma$ varied betwen experiments, $\sigma$ in the range $0.2^{\circ}-0.8^{\circ}$. The target followed the straight line in Figure 5 and 6, starting $20 \mathrm{~km}$ (once $12 \mathrm{~km}$ ) from the origin in northernly direction and moving towards the southeast (on one occasion northeast). Target speed was always $7 \mathrm{~m} / \mathrm{s}$.

The filter was operated under the following conditions. Initial position of the target must be close to the line defined by the first bearing and was assumed to be uniformly distributed between 6 and 60 kilometers along that line. The prior distribution for target speed was Gaussian with mean $7 \mathrm{~m} / \mathrm{s}$ and standard deviation $4 \mathrm{~m} / \mathrm{s}$ (negative values not allowed). The movement of the target could be along any direction in the $\pm 90^{\circ}$ sector around the true one. Initially, any course was as likely as the other. The noise parameters $\sigma$ and $\alpha$ were assigned uniform prior distributions, between $0.1^{\circ}$ and $2.0^{\circ}$ for $\sigma$ and between 0 and 1 for $\alpha$. The smoothing parameter for the density estimate was $\zeta=0.2$. 

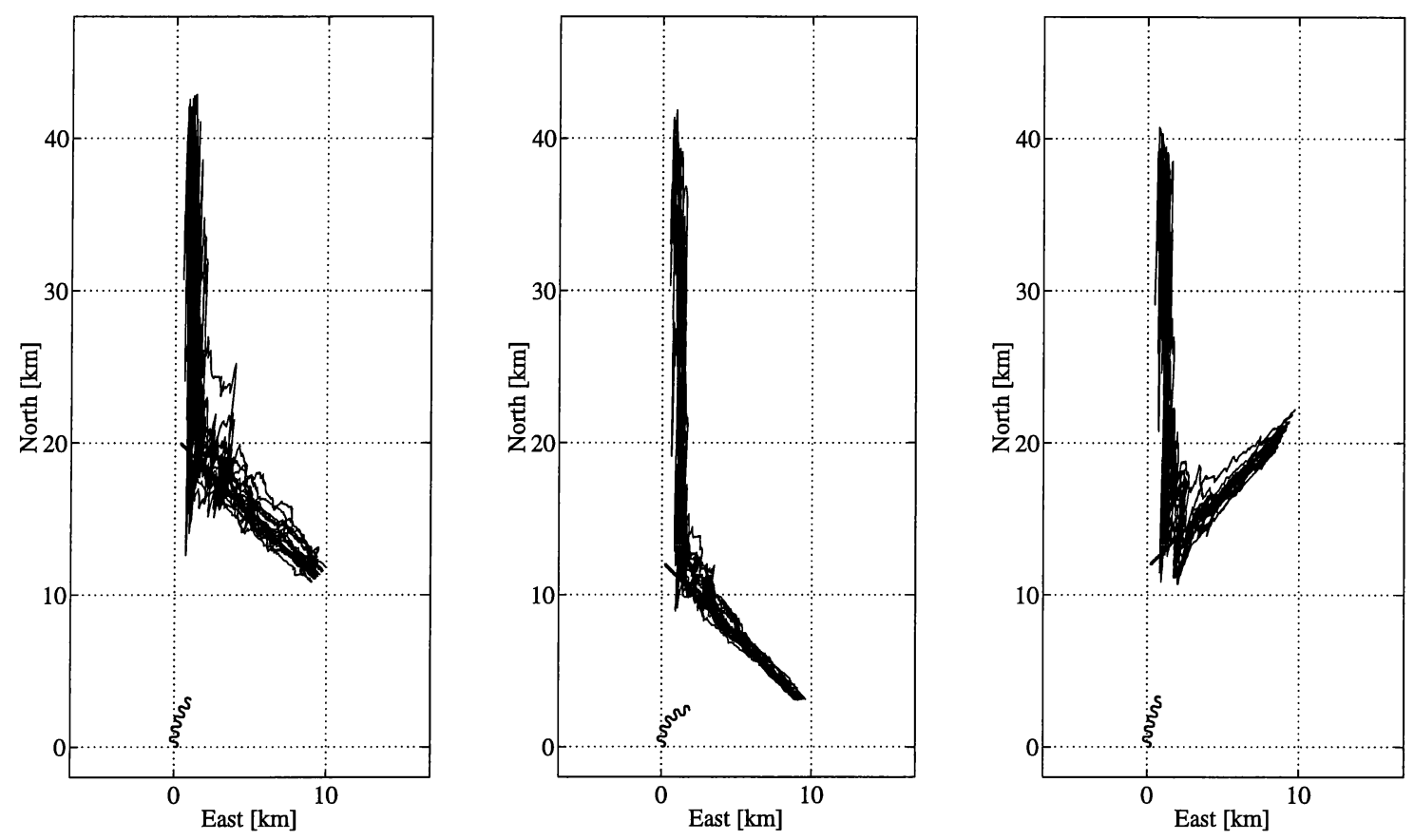

Figure 6: Repeated, filtered estimates of position (different scenarios) for coloured measurement noise $\left(\alpha=0.6\right.$ and $\left.\sigma=0.2^{\circ}\right)$. Twenty observation sequences in each figure, all different. Monte Carlo samples: 5000 in all experiments.

The first round of experiments (Figure 5) examines the variability of the state estimates for a fixed scenario. The measurement noise was white with unknown standard deviation. The true value $\left(0.3^{\circ}\right)$ was estimated on-line which yields a five-dimensional state vector. Figure 5 left displays filtered estimates of position for twenty different realisations of the observation sequence. Each run lasts half an hour and $B=1000$. It takes the filter about 5-10 minutes to close in, and the target is tracked from then on. To single out the contribution of the Monte Carlo uncertainty per se, the other experiments in Figure 5 have been run on the same measurement realisation sequence. Variation between runs is then due to Monte Carlo randomness only. With $B=1000$ (middle figure), the spread is uncomfortably high, although no case of divergence was experienced. When $B$ is raised to 5000 (figure on the right), Monte Carlo uncertainty is much reduced, although higher (perhaps one hundred times) than suggested by the theory in Section 2. The discrepancy is caused by (weak) correlation between individual samples, its origin being the statistical error in the probability density estimates. The correlation applies to all pairs of realisations at $t_{k}$ and is therefore bound to have substantial impact on the variance of the means.

The second round of experiments (Figure 6) were carried out under different scenarios. Measurement noise was now coloured with $\alpha=0.6$ and $\sigma=0.2^{\circ}$. Both parameters were estimated on-line. Twenty runs on different measurement realisation sequences are plotted jointly in 


\begin{tabular}{|l|cccc|cccc|cccc|}
\hline & \multicolumn{3}{|c}{$\sigma=.30$} & \multicolumn{2}{c|}{$\alpha=.00$} & \multicolumn{2}{c|}{$\sigma=.20$} & \multicolumn{2}{c|}{$\alpha=.60$} & \multicolumn{2}{c|}{$\sigma=.80$} & \multicolumn{2}{c|}{$\alpha=.60$} \\
Time & Mean & SD & Mean & SD & Mean & SD & Mean & SD & Mean & SD & Mean & SD \\
3 min. & .31 & .04 & .14 & .08 & .19 & .03 & .52 & .15 & .78 & .10 & .52 & .15 \\
6 min. & .30 & .03 & .10 & .06 & .20 & .02 & .57 & .11 & .79 & .08 & .58 & .10 \\
12 min. & .30 & .02 & .08 & .05 & .20 & .02 & .60 & .09 & .79 & .07 & .60 & .09 \\
\hline
\end{tabular}

Table 1: Mean and standard deviation of estimates of the noise parameters in bearings-only tracking for three sets of true parameters given in the top row. Hundred independent estimates behind each entry, as explained in the text.

each of the three subfigures. Taking $B=1000$ lead to divergence in one of the sixty runs, but $B=5000$ worked well. Estimation is noticeably more accurate at closer distance (middle figure) and somewhat poorer when movement is away from the observer (figure on the right). Raising the noise level from $\sigma=0.2^{\circ}$ to $\sigma=0.3^{\circ}(\alpha=0.6$, as before) gave much larger estimation errors, but the noise parameters themselves could be determined with good accuracy.

This is followed up in the larger experiment in Table 1. The target started $20 \mathrm{~km}$ from the origin as before, but now followed different tracks. Movement was to the north $\left(0^{\circ}\right.$ with the vertical axis) and to northeast $\left(45^{\circ}\right)$, east $\left(90^{\circ}\right)$, southeast $\left(135^{\circ}\right)$ and south $\left(180^{\circ}\right)$. Twenty realisations of each measurement sequence were used in each direction, alltogether one hundred runs. The noise parameters $\sigma$ and $\alpha$ were estimated in each run. Estimation accuracy varied little with direction, and summaries over all directions are therefore presented in Table 1 . Three different sets of true noise parameters were used. The estimates are nicely spread around the true values, after even only three minutes (which correspond to 36 measurements). The bias in the $\alpha$ estimates had almost vanished after six minutes, except when the true $\alpha$ is 0 . (Some bias is then unavoidable, since the true $\alpha$ is on the boundary of the assumed range). Note that estimation errors in $\alpha$ are the same at low and high noise level ( middle and right scenario in Table 1). This phenomenon is well-known in linear models. The general conclusion from Table 1 is that noise parameters can be estimated reliably, even at high noise levels. Errors in these estimates are unlikely to degrade the state estimates much.

\section{Conclusions and discussion}

We have discussed Monte Carlo methods for general, non-linear state estimation. A rejection filter was proposed and its versatility demonstrated through examples. An alternative, similar method is the bootstrap filter, but for reasons given in Section 3.3 we recommend the rejection filter if a suitable $M$ can be found. Implementation of either method demands sampling of $\mathbf{x}_{k}$ given $\mathbf{x}_{k-1}$ (through the system equation) and computation (up to a constant of proportionality) of the likelihood of the observation vector $\mathbf{z}_{k}$ given $\mathbf{x}_{k}$. Many of the programming steps are common to all models, and it is therefore often easy to modify existing programs. For example, measurement saturation could in Section 4.2 be accomodated by changing a few statements, 
and in Section 5 adding system noise or changing the mean fuction $\mu\left(\mathbf{x}_{k}\right)$ in (5.1) would not involve much work.

Ideally Monte Carlo realisations $\mathbf{x}_{k}^{* b}$ should be stochastically independent samples of $\mathbf{x}_{k}$ from its posterior distribution given the data vector $\mathbf{Z}_{k}$. In practice this goal can only be achieved approximately. Statistical errors in the probability density estimates cause the distribution of the Monte Carlo samples to be slightly wrong and introduce weak correlation between samples. The latter lead to underestimation of the variance of the state estimates in Section 4.1 and was also felt in the tracking example in Section 5. These inaccuracies accumulate over time, but presumably not indefinitely. In Section 4.1, where the Kalman filter provided an exact reference, errors turned out to stabilize in the long run. Due to basic properties of Markov processes this must be so under many circumstances, but formal mathematical conditions have not been worked out; see Section 4.1.

Any problem of this kind may be overcome by raising the number of Monte Carlo samples, but then at the expense of more computation. We had to use Monte Carlo sample sizes of several thousand in Section 5, and this is much more than required to control sampling uncertanty per se; see Section 2. It is important to bring Monte Carlo samples sizes down. Not only will this improve the speed of the filters, but it may also open for treatment of state vectors of higher dimension. One line of research is to apply theory and methods from statistical density estimation. We have here used a simple kernel density estimate without giving much thought to how the smoothing parameter $\zeta$ should be chosen. The selection of $\zeta$ was not too critical in the cases tested, but many studies in statistics have been devoted to this issue, and the insight gained there could prove useful. It would be surprising if the kernel metod itself was the most suitable one. For example, although the mean and covariance matrix of the original sample are preserved, the third order moments, biased towards zero by the convolution, are not. More sophisticated techniques could be based on pilot estimates to determine the approximate form of the underlying density; see Silverman (1986). The smoothing parameter $\zeta$ is then found locally at each sample point. There are many other possibilities, and the statistical literature also contains theoretical analyses of density estimation error that could be integrated in the present approach.

Progress in non-linear Monte Carlo filtering is likely to demand close interplay between control engineering and statistics. Scientists from these fields are traditionally separated by a 'cultural gap' which is a brake on interdisciplinary work. (It took 20 years or more for the Kalman filter to be well established in statistics.) But for those managing to bridge the gap, there is scope for future advance in Monte Carlo filtering in several directions.

\section{References}

Alspach, D.L. and H. W. Sorenson (1972). Nonlinear Bayesian Estimation Using Gaussian Sum Approximation. IEEE Transactions on Automatic Control, AC-17, 439-447. 
Borstad, A.J. (1985). Bearings-only target motion analysis estimation characteristics. Control and Computers, 13, 95-101.

Bucy, R.S. and Senne, K.D. (1971). Digital synthesis of non-linear filters. Automatica, 7, 287-298.

Carter, C.A. and Kohn, R. (1994). On Gibbs sampling for state space models. Biometrika, 81, $541-553$.

Devroye, L. (1986). Non-uniform random variate generation. New York: Springer Verlag.

De Jong, P. and Shepard, N. (1995). The simulation smoother for time series models. Biometrika, 82, 339-350.

Efron, B. and Tibshriani, R. (1993). An introduction to the bootstrap. London: Chapman \& Hall.

Eidsvik, J. (1997). Coloured noise in recursive Monte Carlo filters. (In preparation). Master thesis, University of Oslo.

Fröwirth-Schnatter, S. (1995). Bayesian model discrimination and Bayes factors for linear Gaussian state space models. Journal of Royal Statistical Society B, 57, 237-246.

Gordon, N. (1995). A hybrid bootstrap filter for target tracking in clutter. Proceedings of the American Control Conference, Seattle Washington, 628-632.

Gordon, N.J., Salmond, D.J. and Smith, A.F.M. (1993). Novel approach to nonlinear/nonGaussian Bayesian state estimation. IEE Proceedings-F Radar and Signal Processing. 140, $107-113$.

Gordon, N., D. Salmond, and C. Ewing (1995). Bayesian state estimation for tracking and guidance using the bootstrap filter. AIAA Journal of Guidance, Control and Dynamics. 18, 1434-1443.

Kitagawa, G. (1996). Monte Carlo filter and smoother for non-Gaussain, non-linear state space models. Journal of Computational and Graphical statistics. 5, 1-25.

Kramer, S.C. and Sorenson, H.W. (1988). Recursive Bayesian estimation using piece-wise constant approximations. Automatica, 24, 789-801.

Müller, P. (1991). Monte Carlo integration of general dynamic models. Contemporary Mathematics. 115, 145-162.

Scott, D.W. (1992). Multivariate density estimation: Theory, practice and visualization. New York: Wiley. 
Shepard, N. (1994). Partial non-Gaussian state space. Biometrika. 81, 115-131.

Silverman, B. (1986). Density estimation for statistics and data analysis. London: Chapman $\mathcal{E}$ Hall.

Sorenson, H.W. and D. L. Alspach (1971). Recursive Bayesian Estimation Using Gaussian Sums. Automatica. 7, 465-479.

Sverdrup, E. (1967). Laws and chance variations. Amsterdam: North-Holland.

Tanner, M. (1992). Tools for statistical inference. New York: Springer Verlag.

West, M. (1992). Modelling with mixtures. In Bayesian Statistics 4. Bernardo, J.M., Berger, J.O., Dawid, A.P. and Smith, A.F.M. eds. Oxford: Oxford University Press.

\section{A Proofs of results in Section 2}

The basic mathematical condition behind the results in Section 2.1 is that $\mathbf{x}^{* 1}, \ldots, \mathbf{x}^{* B}$ and $\mathbf{x}$ are conditionally independent and identically distributed given $\mathbf{Z}$. This assumption enters all reasoning below. One particular consequence is that $E\left(\mathbf{x}^{* b} \mid \mathbf{Z}\right)=E(\mathbf{x} \mid \mathbf{Z})$, which leads to $E\left(\overline{\mathbf{x}}^{* B} \mid \mathbf{Z}\right)=E(\mathbf{x} \mid \mathbf{Z})$, or, equivalently, $E\left(\overline{\mathbf{x}}^{* B}-\mathbf{x} \mid \mathbf{Z}\right)=0$. Assertion (i) of the theorem now follows by the rule of 'double expectation', i.e. that $E\left(y_{1}\right)=E\left\{E\left(y_{1} \mid y_{2}\right)\right\}$ for any pair of random variables $\left(y_{1}, y_{2}\right)$.

Introduce

$$
\begin{aligned}
& Q_{1}=\left(\overline{\mathbf{x}}^{* B}-\hat{\mathbf{x}}\right)\left(\overline{\mathbf{x}}^{* B}-\hat{\mathbf{x}}\right)^{T} \\
& Q_{2}=(\hat{\mathbf{x}}-\mathbf{x})(\hat{\mathbf{x}}-\mathbf{x})^{T} \\
& Q_{12}=(\hat{\mathbf{x}}-\mathbf{x})\left(\overline{\mathbf{x}}^{* B}-\hat{\mathbf{x}}\right)^{T}
\end{aligned}
$$

Elementary algebra shows that $\left(\overline{\mathbf{x}}^{* B}-\mathbf{x}\right)\left(\overline{\mathbf{x}}^{* B}-\mathbf{x}\right)^{T}=Q_{1}+Q_{2}+2 Q_{12}$, and when taking expectations

$$
P^{B}=E\left(Q_{1}\right)+E\left(Q_{2}\right)+2 E\left(Q_{12}\right) .
$$

We shall prove that

$$
E\left(Q_{1}\right)=B^{-1} P, \quad E\left(Q_{2}\right)=P, \quad E\left(Q_{12}\right)=0,
$$

which yield assertion (ii) of the theorem. First recall that $\hat{\mathbf{x}}=E(\mathbf{x} \mid \mathbf{Z})=E\left(\overline{\mathbf{x}}^{* B} \mid \mathbf{Z}\right)$. Hence $E\left(Q_{1} \mid \mathbf{Z}\right)$ is the conditional covariance matrix of $\overline{\mathbf{x}}^{* B}$ given $\mathbf{Z}$. Since the individual vectors $\mathbf{x}^{* b}$ are conditionally independent, we may use the elementary formula for the covariance matrix of a vector of averages. This yields

$$
E\left(Q_{1} \mid Z\right)=B^{-1} E\left\{\left(\mathbf{x}^{* b}-\hat{\mathbf{x}}\right)\left(\mathbf{x}^{* b}-\hat{\mathbf{x}}\right)^{T} \mid \mathbf{Z}\right\}
$$


But on the right $\mathbf{x}^{* b}$ may be replaced with $\mathbf{x}$ (since their conditional distribution given $\mathbf{Z}$ is the same). Hence

$$
E\left(Q_{1} \mid Z\right)=B^{-1} E\left\{(\mathbf{x}-\hat{\mathbf{x}})(\mathbf{x}-\hat{\mathbf{x}})^{T} \mid \mathbf{Z}\right\}
$$

and by the rule of double expectation $E\left(Q_{1}\right)=B^{-1} E\left\{(\mathbf{x}-\hat{\mathbf{x}})(\mathbf{x}-\hat{\mathbf{x}})^{T}\right\}=B^{-1} P$, as claimed. The second identity, i.e. $E\left(Q_{2}\right)=P$, is valid by definition. Finally, note that

$$
E\left(Q_{12} \mid \mathbf{Z}\right)=E\left\{(\hat{\mathbf{x}}-\mathbf{x})\left(\overline{\mathbf{x}}^{* B}-\hat{\mathbf{x}}\right)^{T} \mid \mathbf{Z}\right\}=E\{(\hat{\mathbf{x}}-\mathbf{x}) \mid \mathbf{Z}\} E\left\{\left(\overline{\mathbf{x}}^{* B}-\hat{\mathbf{x}}\right)^{T} \mid \mathbf{Z}\right\},
$$

since $\overline{\mathbf{x}}^{* B}$ and $\mathbf{x}$ are conditionally independent ( $\hat{\mathbf{x}}$ is a constant for given $\mathbf{Z}$ ). Either of these factors are zero, and $E\left(Q_{12} \mid \mathbf{Z}\right)=0$ which implies $E\left(Q_{12}=0\right.$, as demanded.

The corresponding results for the variance estimates remain. First recall that sample covariance matrices for independently and identically distributed random vectors are unbiased. When applied to $\mathbf{x}^{* 1}, \ldots, \mathbf{x}^{* B}$, this yields

$$
E\left(P^{* B} \mid \mathbf{Z}\right)=E\left\{\left(\mathbf{x}^{* b}-\hat{\mathbf{x}}\right)\left(\mathbf{x}^{* b}-\hat{\mathbf{x}}\right)^{T} \mid \mathbf{Z}\right\}
$$

since $\hat{\mathbf{x}}=E\left(\mathbf{x}^{* b} \mid Z\right)$, as noted earlier. As above, $\mathbf{x}^{* b}$ may be replaced with $\mathbf{x}$. Hence $E\left(P^{* B} \mid \mathbf{Z}\right)=$ $E\left\{(\mathbf{x}-\hat{\mathbf{x}})(\mathbf{x}-\hat{\mathbf{x}})^{T} \mid \mathbf{Z}\right\}$, and, by the rule of double expectation, $E\left(P^{* B}\right)=E\left\{(\mathbf{x}-\hat{\mathbf{x}})(\mathbf{x}-\hat{\mathbf{x}})^{T}\right\}=P$. But by assertion (ii) of the theorem, $P=(1+1 / B)^{-1} P^{B}$, and (2.4) follows.

To prove (2.6) we may invoke the so-called double variance formula, e.g. that $\operatorname{var}\left(y_{1}\right)=$ $\operatorname{var}\left\{E\left(y_{1} \mid y_{2}\right)\right\}+E\left\{\operatorname{var}\left(y_{1} \mid y_{2}\right)\right\}$ for any pair of random variables $\left(y_{1}, y_{2}\right)$ with finite second order moments. On matrix form this yields the decomposition

$$
\operatorname{var}\left(P^{* B}\right)=\operatorname{var}\left\{E\left(P^{* B} \mid \mathbf{Z}\right)\right\}+E\left\{\operatorname{var}\left(P^{* B} \mid \mathbf{Z}\right)\right\},
$$

recalling the definition of var of a matrix as a matrix of variances. We shall show that

$$
E\left(P^{* B} \mid \mathbf{Z}\right)=\hat{P}, \quad \operatorname{var}\left(P^{* B} \mid \mathbf{Z}\right)=B^{-1} \operatorname{var}\left\{(\hat{\mathbf{x}}-\mathbf{x})(\hat{\mathbf{x}}-\mathbf{x})^{T} \mid \mathbf{Z}\right\}+A_{2} B^{-2},
$$

which implies (2.6). The identity on the left is immediate, since we have just proved that $E\left(P^{* B} \mid \mathbf{Z}\right)=E\left\{(\hat{\mathbf{x}}-\mathbf{x})(\hat{\mathbf{x}}-\mathbf{x})^{T} \mid \mathbf{Z}\right\}$, which is $\hat{P}$; see (2.5). The other relationship follows from calculations carried out on in Sverdrup (1967) (p. 155) where a formula for the variance of sample variances of independently and identically distributed observations is derived. 